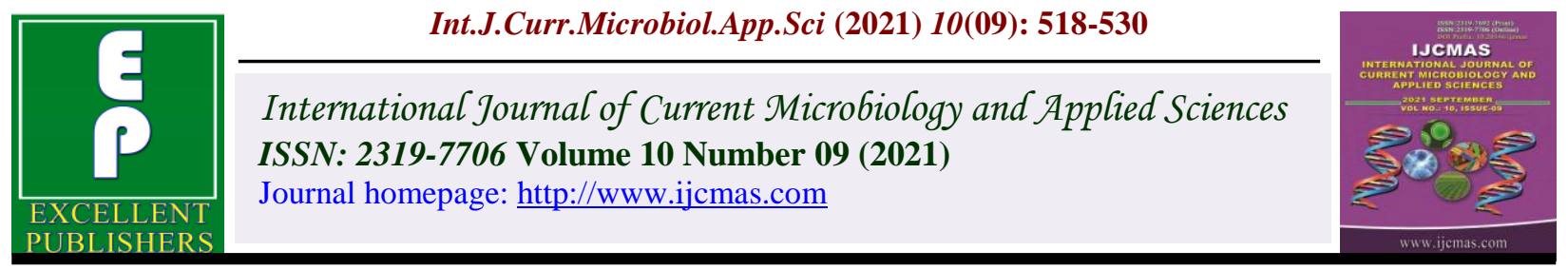

Review Article

https://doi.org/10.20546/ijcmas.2021.1009.060

\title{
Animal Husbandry Mobile Apps in Transformation Livestock Farming
}

\author{
Kakani Vijay Prakash ${ }^{1 *}$, Debasis Ganguli ${ }^{1}$, Arunasis Goswami ${ }^{1}$ and Chanchal Debnath $^{2}$ \\ ${ }^{1}$ Department of Veterinary and Animal Husbandry Extension Education \\ ${ }^{2}$ Department of Veterinary Public Health and Jurisprudence, FVAS, WBUAFS, Kolkata, \\ West Bengal, India \\ *Corresponding author
}

\section{A B S T R A C T}

Keywords

Socio-economic Development, Animal Husbandry system, domestic and global markets

Article Info

Accepted:

20 August 2021

Available Online:

10 September 2021
Mobile apps are potential digital tools which can be effectively utilized to reach Animal Husbandry information to a large number of farmers within a short period of time. They can be used to enhance farm income and productivity through providing correct information, better input and farm management, easy marketing and linkage with government agency for policy support to farmer etc. However, there are challenges like low smart phone penetration rate in rural India, variable internet connectivity, low digital literacy among farmers, limited availability of agricultural information in local languages etc.

\section{Introduction}

Livestock sector plays a multi-faceted role in socio-economic development of rural households and contributes about 4.2 percent to the Gross Domestic Product and 25.6 percent to the Agricultural Gross Domestic Product in the country. Empirical evidences indicate that livestock is an important component of the Animal Husbandry system, providing an additional source of income and nutritional cover to a large section of the rural population, particularly the disadvantaged and poor households (Rao et al., 2003; Birthal and Ali, 2005; Ravikumar and Chander, 2006;
Singh et al., 2007). The distribution of livestock, as a liquid asset to poor families, is more egalitarian as compared to land (Taneja and Birthal, 2004). However, the recent trend in livestock sector growth suggests that in order to meet the emerging demand for livestock based products, both in domestic and global markets, there is a need to reorient the production system by enhancing the efficiency and creating quality consciousness. Adhiguru et al., (2009) argued that farmers are not only looking for various information sources for carrying out their production and marketing tasks efficiently but also for ensuring delivery of safe and quality products to the consumers. 
ICT based information delivery to livestock sector can significantly improve the quality of decision-making in livestock farming system (Tiwari et al., 2010).

Among ICTs, there has been increasing use of mobile phones which is changing the agricultural communication process. The introduction of mobile phones has resulted in new services and applications. There are an increasing number of mobile apps providing access to Animal Husbandry and allied sector information. A mobile application is software on a mobile phone handset or tablet computer that enables a user to access specific information; make payments and other transactions; send messages; etc. The application (app) is downloaded (for free or for payment) from a wireless network from an online store and may require a live connection to function effectively. The main advantages of mobile apps for farmers are, easy to access information on farmers mobile. The information is stored in the mobile handset itself for easy access. The scope of mobile apps in offering EAS (Extension and Advisory service) is tremendous during COVID-19 pandemic situation. This will enable the farmers for self management of livestock in such disaster situations.

Government of India has launched a number of web and mobile based applications for dissemination of information on Animal Husbandry related activities, free of cost, for the benefit of farmers and other stakeholders. There are apps also developed by Veterinary institutions, private sector, NGOs. These apps are disseminating information from livestock research and extension to farmers and other stakeholders and facilitating exchange of information among stakeholders. The documentation of these mobile apps and evaluating their content and features is of immense importance for the farmers as it will provide guidance in choosing suitable app for getting need based reliable information for livestock farming. This will also assist the mobile app developers in designing new app or in modifying and improving their exiting mobile apps.

Farmers can easily access these portals through smart mobile phones. Through internet, they can get the information pertaining to current market prices and many crop related facts that can provide them more benefits. Worldwide most of the farmers, even in remote places have mobile phones. They can be equipped with targeted agricultural advice through text and voice message even without internet access. These timely delivered advisories at low cost provide improvement in farmers' practices for increasing agricultural income. Mobile apps also provide help to Government and private sector for distributing subsidies to farmers or managing the inventories of emergency input stocks in government storage facilities. It encouraged more data sharing between the private and public sectors in service of the public good. Country like India, where diversification in languages exists among farming community; regionally suitable mobile apps with information on soil, climate, nutrient management and input price could be effectively helpful (Inwood \& Dale, 2019). Youth farmers' generations are very much stick to their mobile phones and therefore, information can very quickly reach through digital applications (Rose et al., 2016).

\section{Widely used Mobile Apps in the field of Animal Husbandry}

Mobile apps for Animal Husbandry help in acquiring knowledge and decision making. A range of mobile apps have been developed for the benefit of the farmers for improving information transfer efficiency and significantly impact Livestock business. This study categorized some of the commonly 
available Animal Husbandry apps for different purposes such as

Mobile Apps were categorized into the following in Animal Husbandry

Central Government \& State Government developed Apps

Indian Council of Agriculture Research (ICAR) developed Apps

State Agricultural Universities (SAU's) developed Apps

Dairy Co-operatives \& Private Organizations developed Apps

Following are the major advantages of using mobile apps in Animal Husbandry sector

Provides advisories through text and video messaging services.

Access to information on Management, Nutrition, etc which directly affect agricultural decision making.

Market intelligence through providing data on prices, quality and quantity of arrival of various agricultural commodities at different markets across the country.

Online monitoring and management of /livestock/poultry/fisheries etc is possible through mobile apps.

Provision of feedback from farmers and other stakeholders in different fields of agriculture.

Information on important machineries and equipments are easily accessible using these apps.
Mobile apps can be utilized for delivering services offered by Government to farmers in the form of inputs and subsidy distribution.

It can be used in management of irrigation systems in large field, sensor based farming, identification of different soil types etc.

It facilitates effective farm management by recording data, analyzing it and giving suitable recommendation for different enterprises.

\section{Challenges of Using Mobile Apps in India}

It requires skilled manpower to operate mobile apps for which digital literacy is must.

Diversity in language across the country makes it difficult to make apps in local languages.

The content requires translation at many stages which may affects its quality and reduce acceptability among farming community.

Better internet speed is required for using mobile apps. Many times in rural area, internet connection and speed is a major issue which affects mobile application services.

Farmers are not adequately equipped with required knowledge \& skill to use mobile apps.

Many mobile apps are charging money for its use, therefore farmer may not afford paid services through these apps.

Lower adoption and use of mobile apps by farmer is a huge challenge and government must enhance digital literacy in order to resolve it. 
Table.1 Central Government \& State Government developed Apps

\begin{tabular}{|c|c|c|c|c|}
\hline & S. No & App Name & LOGO & LINK \\
\hline \multirow{9}{*}{$\begin{array}{c}\text { Ministry of } \\
\text { Fisheries } \\
\text { Animal } \\
\text { Husbandry and } \\
\text { Dairying, } \\
\text { New Delhi - } \\
\text { National Dairy } \\
\text { Development } \\
\text { Board, Gujarat }\end{array}$} & 1. & e-GOPALA & 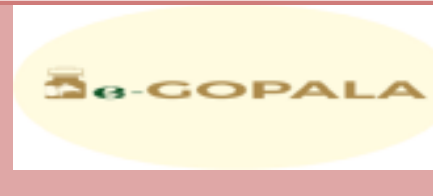 & $\begin{array}{l}\text { https://play.google.com/store } \\
\text { lapps/details?id=coop.nddb. } \\
\text { pashuposhan\&hl=en_IN\&gl } \\
=U S\end{array}$ \\
\hline & 2. & NDDB AGR & & $\begin{array}{c}\text { https://play.google.com/store } \\
\text { /apps/details?id=com.iviewl } \\
\text { abs.nddbar\&hl=en_IN\&gl= } \\
\text { US }\end{array}$ \\
\hline & 3. & $\begin{array}{l}\text { Dairy Husbandry } \\
\text { Practices (Hi) }\end{array}$ & Ex & $\begin{array}{l}\text { https://play.google.com/store } \\
\text { /apps/details?id=coop.nddb. } \\
\text { guidetoanimalhusbandry_hi } \\
\text { ndi\&hl=en_IN\&gl=US }\end{array}$ \\
\hline & 4. & $\begin{array}{l}\text { Dairy Husbandry } \\
\text { Practices }\end{array}$ & & $\begin{array}{l}\text { https://play.google.com/store } \\
\text { /apps/details?id=coop.nddb. } \\
\text { guidetoanimalhusbandry\&hl } \\
\quad=e n \_I N \& g l=U S\end{array}$ \\
\hline & 5. & Journey of Milk & & $\begin{array}{c}\text { https://play.google.com/store } \\
\text { lapps/details?id=com.nddb.j } \\
\text { om\&hl=en_IN\&gl=US }\end{array}$ \\
\hline & 6. & $\begin{array}{l}\text { Story Of Milk A } \\
\text { Cow's Tale (en) }\end{array}$ & & $\begin{array}{c}\text { https://play.google.com/store } \\
\text { /apps/details?id=org.nddb\& } \\
\text { hl=en_IN\&gl=US }\end{array}$ \\
\hline & 7. & $\begin{array}{l}\text { Story Of Milk A } \\
\text { Cow's Tale (hi) }\end{array}$ & & $\begin{array}{c}\text { https://play.google.com/store } \\
\text { lapps/details?id=org.nddb.st } \\
\text { oryofcow.hindi\&hl=en_IN\& } \\
\text { gl=US }\end{array}$ \\
\hline & 8. & $\begin{array}{l}\text { INAPH In- } \\
\text { Breeding } \\
\text { Check }\end{array}$ & & $\begin{array}{c}\text { https://play.google.com/store } \\
\text { /apps/details?id=coop.nddb. } \\
\text { inaph.in_breeding_check\&hl } \\
=\text { en_IN\&gl=US }\end{array}$ \\
\hline & 9. & $\begin{array}{l}\text { Pashu Poshan } \\
\text { Kisan Rath }\end{array}$ & & $\begin{array}{c}\text { https://play.google.com/store } \\
\text { lapps/details?id=coop.nddb. } \\
\text { pashu_poshan\&hl=en_IN\&g } \\
l=U S\end{array}$ \\
\hline $\begin{array}{c}\text { National } \\
\text { Information } \\
\text { Centre, New } \\
\text { Delhi }\end{array}$ & 1. & Kisan Rath & & $\begin{array}{c}\text { https://play.google.com/store } \\
\text { lapps/details?id=com.velocis } \\
\text {.app.kishan.vahan\&hl=en_I } \\
\text { N\&gl=US }\end{array}$ \\
\hline
\end{tabular}


Table.2 Indian Council of Agriculture Research developed (ICAR) Apps

\begin{tabular}{|c|c|c|c|c|}
\hline Institute & S. No & App Name & LOGO & LINK \\
\hline \multirow{2}{*}{$\begin{array}{l}\text { Indian Council } \\
\text { of Agriculture } \\
\text { Research, } \\
\text { New Delhi }\end{array}$} & 1. & KISAAN & & $\begin{array}{c}\text { https://play.google.com/store/ap } \\
\text { ps/details?id=net.mobile.icar.kis } \\
\text { aan }\end{array}$ \\
\hline & 2. & ICAR Technologies & & $\begin{array}{c}\text { https://play.google.com/store/ap } \\
\text { ps/details?id=gov.krishi.icar.tec } \\
\text { hnologyrepository }\end{array}$ \\
\hline \multirow{4}{*}{$\begin{array}{c}\text { Central } \\
\text { Institute for } \\
\text { Research on } \\
\text { Goats, } \\
\text { Makhdoom }\end{array}$} & 1. & $\begin{array}{c}\text { Bakrimitra (ICAR - } \\
\text { CIRG) }\end{array}$ & 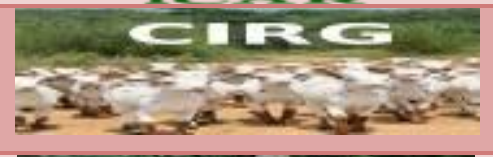 & $\begin{array}{c}\text { https://play.google.com/storelap } \\
\text { ps/details?id=com.cirg.bakrimit } \\
\text { ra\&hl=en_IN\&gl=US }\end{array}$ \\
\hline & 2. & $\begin{array}{c}\text { Bakrimitra (ICAR - } \\
\text { CIRG) }\end{array}$ & & $\begin{array}{c}\text { https://play.google.com/store/ap } \\
\text { ps/details?id=com.cirg.goatfar } \\
\text { ming\&hl=ta\&gl=US }\end{array}$ \\
\hline & 3. & $\begin{array}{l}\mathrm{AI} \text { in Goats(बकरी } \\
\text { गर्भाधान सेतु) }\end{array}$ & & $\begin{array}{l}\text { https://play.google.com/store/ap } \\
\text { ps/details?id=com.goat_incimat } \\
\text { ion\&hl=en_US\&gl=US }\end{array}$ \\
\hline & 4. & $\begin{array}{l}\text { CIRG - Goat Farming } \\
\text { (बकरी पालन / } \\
\text { வெள்ளாடு/ ఆడు) }\end{array}$ & & $\begin{array}{c}\text { https://play.google.com/store/ap } \\
\text { ps/details?id=com.cirg.goatpro } \\
\text { ducts\&hl=ta\&gl=US }\end{array}$ \\
\hline $\begin{array}{l}\text { Directorate of } \\
\text { Poultry } \\
\text { Research, } \\
\text { Hyderabad }\end{array}$ & 1. & ICAR DPR App & & $\begin{array}{l}\text { https://play.google.com/store/ap } \\
\text { ps/details?id=com.webfarmatics } \\
\text {.icar\&hl=en_IN\&gl=US }\end{array}$ \\
\hline \multirow{3}{*}{$\begin{array}{c}\text { National } \\
\text { Institute on } \\
\text { Veterinary } \\
\text { Epidemiology } \\
\text { and Disease } \\
\text { Informatics, } \\
\text { Bangalore }\end{array}$} & 1. & AINP-GIP & & $\begin{array}{c}\text { https://play.google.com/store/ap } \\
\text { ps/details?id=info.nivedi.gip\&hl } \\
\text { =en_IN\&gl=US }\end{array}$ \\
\hline & 2. & Leptospirosis & & $\begin{array}{l}\text { https://play.google.com/store/ap } \\
\text { ps/details?id=com.nivedi.leptos } \\
\text { pirosis\&hl=en_IN\&gl=US }\end{array}$ \\
\hline & 3. & LDF & & $\begin{array}{c}\text { https://play.google.com/storelap } \\
\text { ps/details?id=info.androidhive.l } \\
\text { df\&hl=en_IN\&gl=US }\end{array}$ \\
\hline \multirow{2}{*}{$\begin{array}{c}\text { Central } \\
\text { Institute of } \\
\text { Research on } \\
\text { Buffaloes, } \\
\text { Hisar }\end{array}$} & 1. & $\begin{array}{l}\text { ICAR-CIRB Bhains } \\
\text { Janan (Buffalo } \\
\text { Reproduction) App }\end{array}$ & II IER IPE & $\begin{array}{l}\text { https://play.google.com/storelap } \\
\text { ps/details?id=com.cirb\&hl=en_ } \\
\text { IN\&gl=US }\end{array}$ \\
\hline & 2. & $\begin{array}{l}\text { ICAR-CIRB Bhains } \\
\text { Poshahar (Buffalo } \\
\text { Nutrition) App }\end{array}$ & & $\begin{array}{l}\text { https://play.google.com/store/ap } \\
\text { ps/details?id=com.cirb.buffalop } \\
\text { oshahar\&hl=en_IN\&gl=US }\end{array}$ \\
\hline
\end{tabular}




\begin{tabular}{|c|c|c|c|c|}
\hline & 3. & ICAR-CIRB Bufelth & 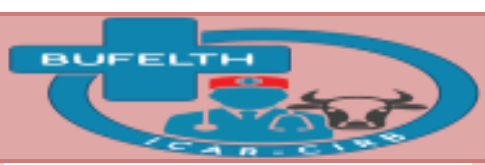 & $\begin{array}{c}\text { https://play.google.com/storelap } \\
\text { ps/details?id=com.cirb.buffhealt } \\
\text { h\&hl=en_IN\&gl=US }\end{array}$ \\
\hline \multirow{12}{*}{$\begin{array}{l}\text { Indian } \\
\text { Veterinary } \\
\text { Research } \\
\text { Institute, } \\
\text { Izathnagar }\end{array}$} & 1. & $\begin{array}{l}\text { IVRI-Pig Farming } \\
\text { App(शूकर पालन) }\end{array}$ & & $\begin{array}{c}\text { https://play.google.com/store/ap } \\
\text { ps/details?id=com.icar.ivri.pig } \\
\text { app\&hl=hi\&gl=US }\end{array}$ \\
\hline & 2. & $\begin{array}{c}\text { IVRI- Waste } \\
\text { Management Guide } \\
\text { App }\end{array}$ & & $\begin{array}{l}\text { https://play.google.com/store/ap } \\
\text { ps/details?id=com.icar.ivri.iasri } \\
\text {.wmapp\&hl=en_IN\&gl=US }\end{array}$ \\
\hline & 3. & $\begin{array}{l}\text { IVRI - Artificial } \\
\text { Insemination App }\end{array}$ & & $\begin{array}{c}\text { https://play.google.com/storelap } \\
\text { ps/details?id=com.ivri.iasri.aia } \\
\text { pp\&hl=en_IN\&gl=US }\end{array}$ \\
\hline & 4. & $\begin{array}{l}\text { IVRI- Biosecurity } \\
\text { and Biosafety (Jaiv } \\
\text { Suraksha) App }\end{array}$ & & $\begin{array}{l}\text { https://play.google.com/store/ap } \\
\text { ps/details?id=com.icar.ivri.iasri } \\
\text {.biosecuriy\&hl=en\&gl=US }\end{array}$ \\
\hline & 5. & $\begin{array}{l}\text { IVRI-Dairy Manager } \\
\text { App (डेयरी मैनेजर) }\end{array}$ & & $\begin{array}{c}\text { https://play.google.com/store/ap } \\
\text { ps/details?id=com.ivri.iasri.dma } \\
\text { pp\&hl=hi\&gl=US }\end{array}$ \\
\hline & 6. & $\begin{array}{l}\text { IVRI-Disease Control } \\
\text { (रोग नियंत्रण एप्प) } \\
\text { App }\end{array}$ & Disease Control App & $\begin{array}{c}\text { https://play.google.com/store/ap } \\
\text { ps/details?id=com.icar.ivri.iasri } \\
\text { diseasecontrolapp\&hl=hi\&gl= } \\
\text { US }\end{array}$ \\
\hline & 7. & $\begin{array}{c}\text { IVRI - Landlly Pig } \\
\text { App }\end{array}$ & & $\begin{array}{l}\text { https://play.google.com/store/ap } \\
\text { ps/details?id=com.ivri.iasri.lan } \\
\text { dlypig\&hl=en_IN\&gl=US }\end{array}$ \\
\hline & 8. & $\begin{array}{l}\text { IVRI-Animal } \\
\text { Reproduction } \\
\text { App(पशु प्रजनन) }\end{array}$ & ANIMAL REPRODUCTIONN & $\begin{array}{l}\text { https://play.google.com/storelap } \\
\text { ps/details?id=com.mraaapsjd.ak } \\
\text { mu.pasujanapp\&hl=hi\&gl=US }\end{array}$ \\
\hline & 9. & $\begin{array}{l}\text { बाजरा संकर नेपियर(B- } \\
\text { N Hybrid): कम लागत } \\
\text { पर हरा चारा }\end{array}$ & & $\begin{array}{c}\text { https://play.google.com/store/ap } \\
\text { ps/details?id=in.nic.ivri.agapp } \\
\text { \&hl=hi\&gl=US }\end{array}$ \\
\hline & 10. & $\begin{array}{l}\text { IVRI - Pig Ration } \\
\text { (शूकर राशन ) App }\end{array}$ & Pig Ration & $\begin{array}{c}\text { https://play.google.com/store/ap } \\
\text { ps/details?id=icar.iasri.ivri.pigr } \\
\text { ation\&hl=hi\&gl=US }\end{array}$ \\
\hline & 11. & $\begin{array}{l}\text { IVRI-Technologies \& } \\
\text { Services App } \\
\text { तकनीकी एवम सेवाएं }\end{array}$ & IVRI- Technologies and Services & $\begin{array}{c}\text { https://play.google.com/store/ap } \\
\text { ps/details?id=com.icar.ivri.iasri } \\
\text {.ivritechnologiesandservicesapp } \\
\text { \&hl=hi\&gl=US }\end{array}$ \\
\hline & 12. & $\begin{array}{c}\text { IVRI-Vaccination } \\
\text { Guide App(टीकाकरण } \\
\text { गाइड) }\end{array}$ & Vaccination Guide & $\begin{array}{l}\text { https://play.google.com/store/ap } \\
\text { ps/details?id=com.icar.ivri.iasri } \\
\text {.vcguideapp\&hl=hi\&gl=US }\end{array}$ \\
\hline
\end{tabular}




\begin{tabular}{|c|c|c|c|c|}
\hline & 13. & $\begin{array}{l}\text { IVRI-Veterinary } \\
\text { Clinical Care App } \\
\text { पशु चिकित्सा सेवा }\end{array}$ & Veterinary Clin & $\begin{array}{l}\text { https://play.google.com/store/ap } \\
\text { ps/details?id=com.icar.ivri.iasri } \\
\text {.veterinaryclinicalcareapp } \& \text { hl }= \\
\text { hi \&gl=US }\end{array}$ \\
\hline & 14. & $\begin{array}{l}\text { IVRI-Zoonoses App } \\
\text { (ज़ूनोसेस एप्प) }\end{array}$ & & $\begin{array}{c}\text { https://play.google.com/store/ap } \\
\text { ps/details?id=com.icar.ivri.iasri } \\
\text {.zoonosesapp\&hl=en_US\&gl= } \\
\text { US }\end{array}$ \\
\hline & 15. & $\begin{array}{l}\text { पशुओं में परजीवी } \\
\text { नियंत्रण }\end{array}$ & 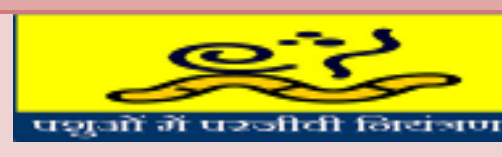 & $\begin{array}{c}\text { https://play.google.com/store/ap } \\
\text { ps/details?id=in.res.ivri.parainf } \\
\text { o\&hl=hi\&gl=US }\end{array}$ \\
\hline & 16. & $\begin{array}{l}\text { Organic Livestock } \\
\text { Farming }\end{array}$ & & $\begin{array}{c}\text { https://play.google.com/store/ap } \\
\text { ps/details?id=in.ivri.res.olfapp } \\
\& h l=\text { en_IN\&gl=US }\end{array}$ \\
\hline \multirow{2}{*}{$\begin{array}{c}\text { ICAR-Indian } \\
\text { Statistical } \\
\text { Research } \\
\text { Institute, } \\
\text { New Delhi }\end{array}$} & 1. & KVK Mobile App & & $\begin{array}{c}\text { https://play.google.com/store/ap } \\
\text { ps/details?id=com.icar.iasri.kvk } \\
\text { app\&hl=en_IN\&gl=US }\end{array}$ \\
\hline & 2. & NIBLD & & 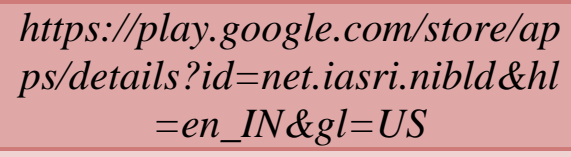 \\
\hline $\begin{array}{l}\text { Central Sheep } \\
\text { and Wool } \\
\text { Research } \\
\text { Institute, } \\
\text { Avikanagar }\end{array}$ & 1. & Avimitra & & $\begin{array}{l}\text { https://play.google.com/store/ap } \\
\text { ps/details?id=com.variablesoft. } \\
\text { avimitra\&hl=en_IN\&gl=US }\end{array}$ \\
\hline $\begin{array}{c}\text { KVK Karur, } \\
\text { Karur }\end{array}$ & 1. & Cattle Fertility App & & $\begin{array}{c}\text { https://play.google.com/store/ap } \\
\text { ps/details?id=com.designexpert } \\
\text { s.cow_fertility\&hl=en\&gl=US }\end{array}$ \\
\hline $\begin{array}{c}\text { National } \\
\text { Research } \\
\text { Centre on Meat, } \\
\text { Hyderabad }\end{array}$ & 1. & Meat App & & $\begin{array}{c}\text { https://play.google.com/store/ap } \\
\text { ps/details?id=in.gov.icar.nrcme } \\
\text { at\&hl=en_IN\&gl=US }\end{array}$ \\
\hline $\begin{array}{l}\text { National } \\
\text { Research } \\
\text { Centre on } \\
\text { Mithun, } \\
\text { Nagaland }\end{array}$ & 1. & Mithun-mitra App & 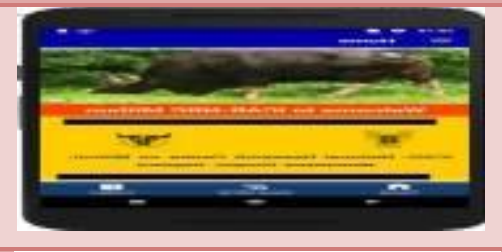 & $\begin{array}{l}\text { https://appagg.com/android/edu } \\
\text { cation/mithun-mitra- } \\
\text { 34175867.html?hl=en }\end{array}$ \\
\hline $\begin{array}{c}\text { National } \\
\text { Institute on } \\
\text { Animal } \\
\text { Nutrition and }\end{array}$ & 1. & Feed Chart App & & $\begin{array}{c}\text { https://play.google.com/storelap } \\
\text { ps/details?id=com.borne.root.ni } \\
\text { anp_feedchart\&hl=en_IN\&gl= } \\
\text { US }\end{array}$ \\
\hline
\end{tabular}




\begin{tabular}{|c|c|c|c|c|}
\hline $\begin{array}{l}\text { Physiology, } \\
\text { Bangalore }\end{array}$ & 2. & $\begin{array}{l}\text { ICAR-NIANP Smart } \\
\text { Tools }\end{array}$ & PIn & $\begin{array}{l}\text { https://apkpure.com/icar-nianp- } \\
\text { smart-tools/com.feedformulation }\end{array}$ \\
\hline $\begin{array}{c}\text { National } \\
\text { Institute on } \\
\text { High Security } \\
\text { Animal } \\
\text { Diseases, } \\
\text { Bhopal }\end{array}$ & 1. & $\begin{array}{l}\text { Bird Flu se Suraksha } \\
\text { App }\end{array}$ & & $\begin{array}{c}\text { https://play.google.com/storelap } \\
\text { ps/details?id=com.action.birdflu } \\
\& h l=\text { en_IN\&gl=US }\end{array}$ \\
\hline \multirow{3}{*}{$\begin{array}{c}\text { Indian } \\
\text { Grassland and } \\
\text { Forage } \\
\text { Research } \\
\text { Institute - } \\
\text { All India } \\
\text { Coordinated } \\
\text { Research } \\
\text { Project, Jhansi }\end{array}$} & 1. & $\begin{array}{l}\text { Fodder \& Range } \\
\text { Grasses }\end{array}$ & & $\begin{array}{c}\text { https://play.google.com/store/ap } \\
\text { ps/details?id=com.frgapp.app \& } \\
\text { hl=en_IN\&gl=US }\end{array}$ \\
\hline & 2. & Forage India & & $\begin{array}{l}\text { https://play.google.com/store/ap } \\
\text { ps/details?id=inforage_india.re } \\
\text { s.httpaicrponforagecrops.forage } \\
\quad \text { india\&hl=en_IN\&gl=US }\end{array}$ \\
\hline & 3. & Forage Seed & & $\begin{array}{l}\text { https://play.google.com/store/ap } \\
\text { ps/details?id=com.aicrpforages } \\
\text { eed.app\&hl=en_IN\&gl=US }\end{array}$ \\
\hline $\begin{array}{c}\text { National } \\
\text { Bureau of } \\
\text { Animal Genetic } \\
\text { Resources of } \\
\text { India, Karnal }\end{array}$ & 1. & $\begin{array}{l}\text { Animal Genetic } \\
\text { Resources of India } \\
\text { (AGRI-IS) App }\end{array}$ & & $\begin{array}{c}\text { https://play.google.com/store/ap } \\
\text { ps/details?id=localhost.angrapp } \\
\& h l=e n \_I N \& g l=U S\end{array}$ \\
\hline $\begin{array}{l}\text { Central Avian } \\
\text { Research } \\
\text { Institute, } \\
\text { Izathnagar }\end{array}$ & 1. & ICAR CARI App & & $\begin{array}{l}\text { https://play.google.com/store/ap } \\
\text { ps/details?id=com.cari.egg }\end{array}$ \\
\hline $\begin{array}{c}\text { National } \\
\text { Research } \\
\text { Centre on } \\
\text { Equine, Hisar }\end{array}$ & 1. & Infoequine App & & $\begin{array}{l}\text { https://play.google.com/store/ap } \\
\text { ps/details?id=info.ncre\&hl=en_ } \\
\text { IN\&gl=US }\end{array}$ \\
\hline \multirow[t]{3}{*}{$\begin{array}{c}\text { National } \\
\text { Research } \\
\text { Centre on Pig, } \\
\text { Assam }\end{array}$} & 1. & Swine App & & $\begin{array}{l}\text { https://play.google.com/store/ap } \\
\text { ps/details?id=com.icar.actech.s } \\
\text { wineapp\&hl=en_IN\&gl=US }\end{array}$ \\
\hline & 2. & $\begin{array}{l}\text { Swine Summer Stress } \\
\text { App }\end{array}$ & & $\begin{array}{l}\text { https://play.google.com/store/ap } \\
\text { ps/details?id=com.kgec.swinesu } \\
\text { mmerstress\&hl=en_IN\&gl=US }\end{array}$ \\
\hline & 3. & $\begin{array}{l}\text { African Swine Fever } \\
\text { Hindi App }\end{array}$ & & $\begin{array}{c}\text { https://play.google.com/store/ap } \\
\text { ps/details?id=com.kgecicar.afri } \\
\text { canswinefeverhindi\&hl=en\&gl= } \\
\text { US }\end{array}$ \\
\hline
\end{tabular}




\begin{tabular}{|c|c|c|c|c|}
\hline & 4. & $\begin{array}{c}\text { African Swine Fever } \\
\text { App }\end{array}$ & $\begin{array}{l}\text { https://play.google.com/store/ap } \\
\text { ps/details?di=com.kgec.africans } \\
\text { winefever\&hl=en_IN\&gl=US }\end{array}$ \\
\hline $\begin{array}{c}\text { National Dairy } \\
\text { Research } \\
\text { Institute, } \\
\text { Karnal }\end{array}$ & 1. & $\begin{array}{c}\text { Dairy Animal } \\
\text { Reproduction }\end{array}$ & $\begin{array}{c}\text { https://play.google.com/store/ap } \\
\text { s/details?did=ndri.drnishant.ani } \\
\text { malreprodution\&hl=en_IN }\end{array}$ \\
\hline
\end{tabular}

Table.3 State Agricultural Universities developed (SAU's) Apps

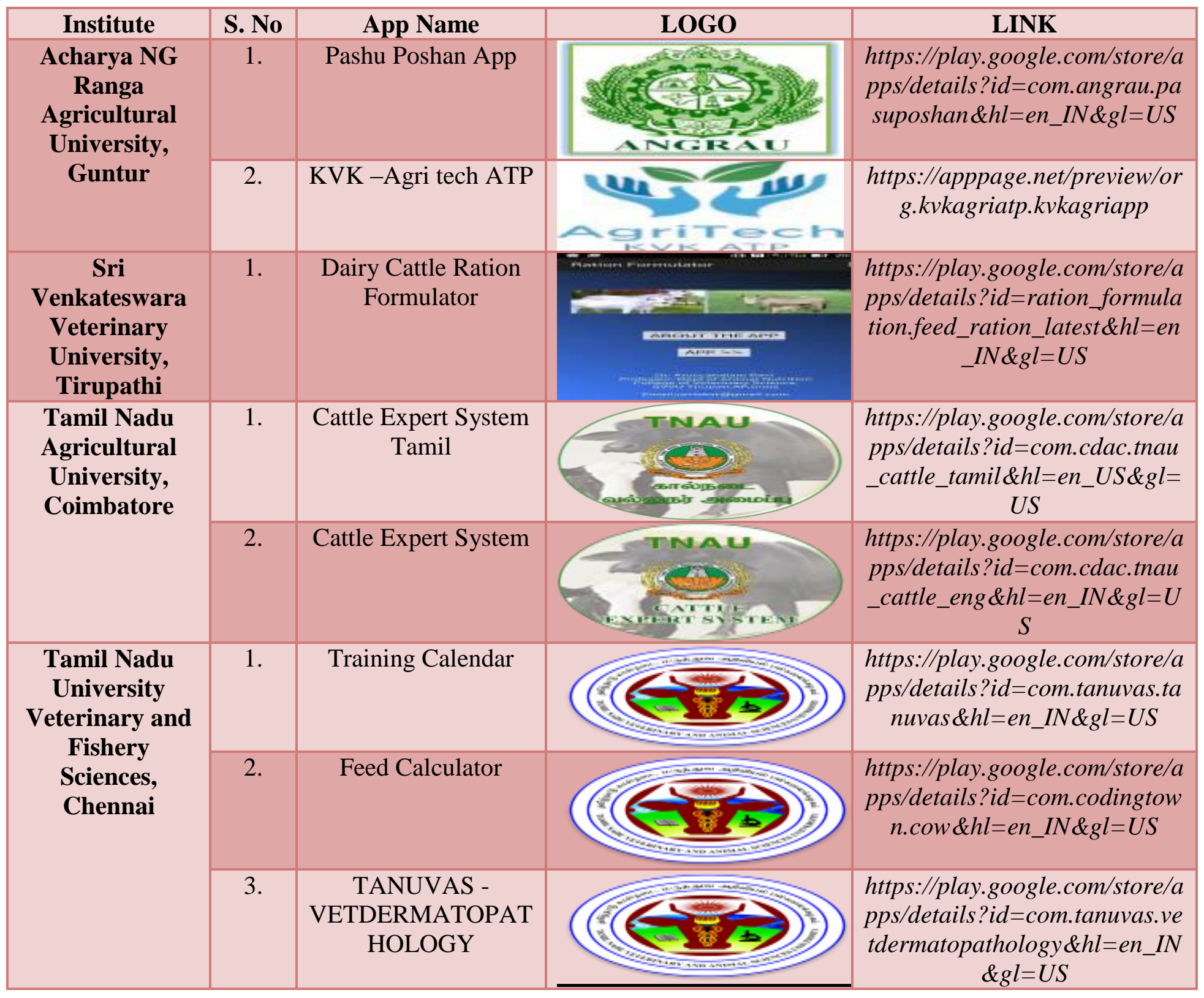




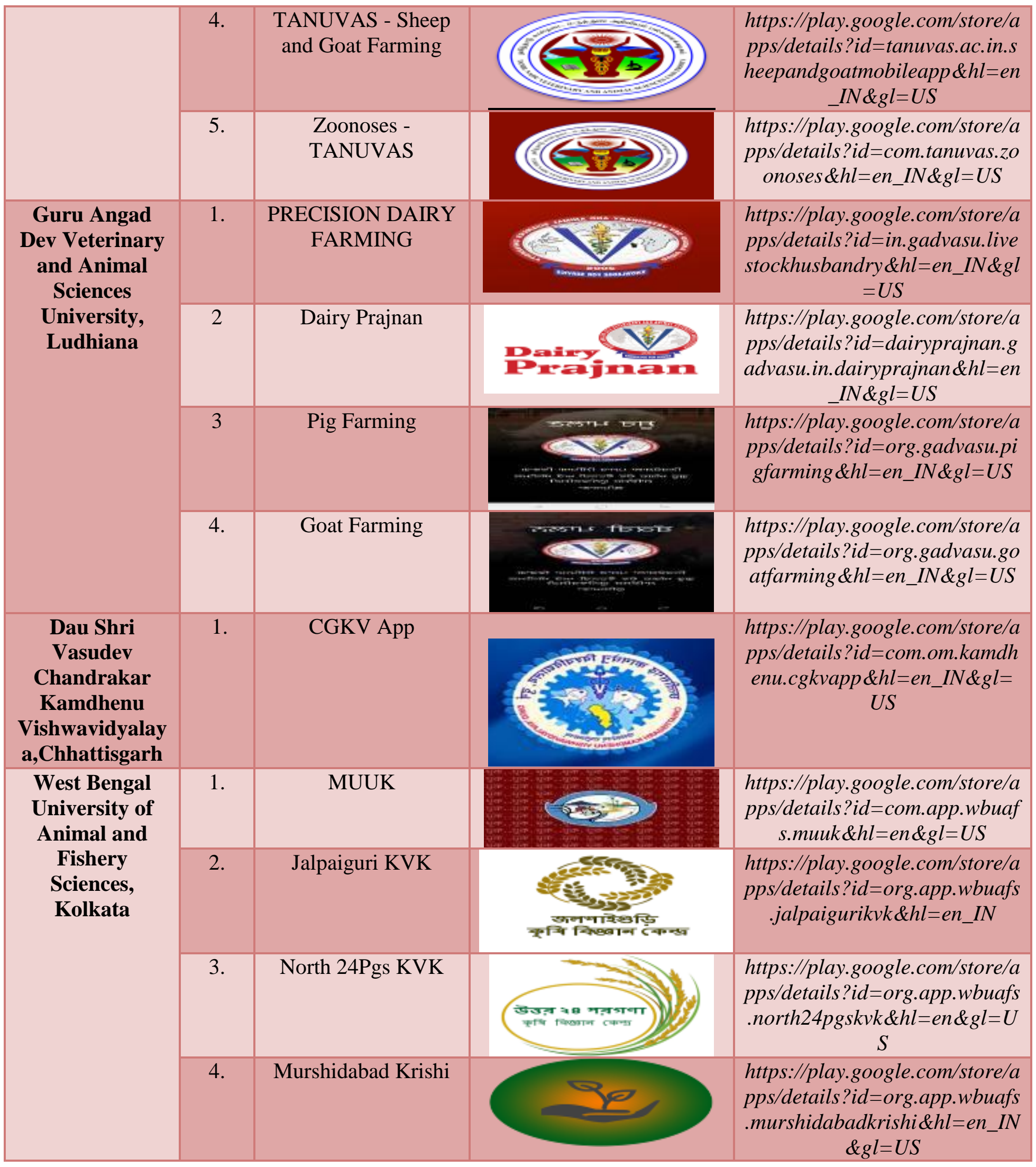




\begin{tabular}{|c|c|c|c|c|}
\hline $\begin{array}{c}\text { Sri Karan } \\
\text { Narendra } \\
\text { Agriculture } \\
\text { University, } \\
\text { Jobner }\end{array}$ & 1. & KVK Ajmer & & \\
\hline
\end{tabular}

Table.4 Dairy Co-operatives \& Private Organizations developed Apps

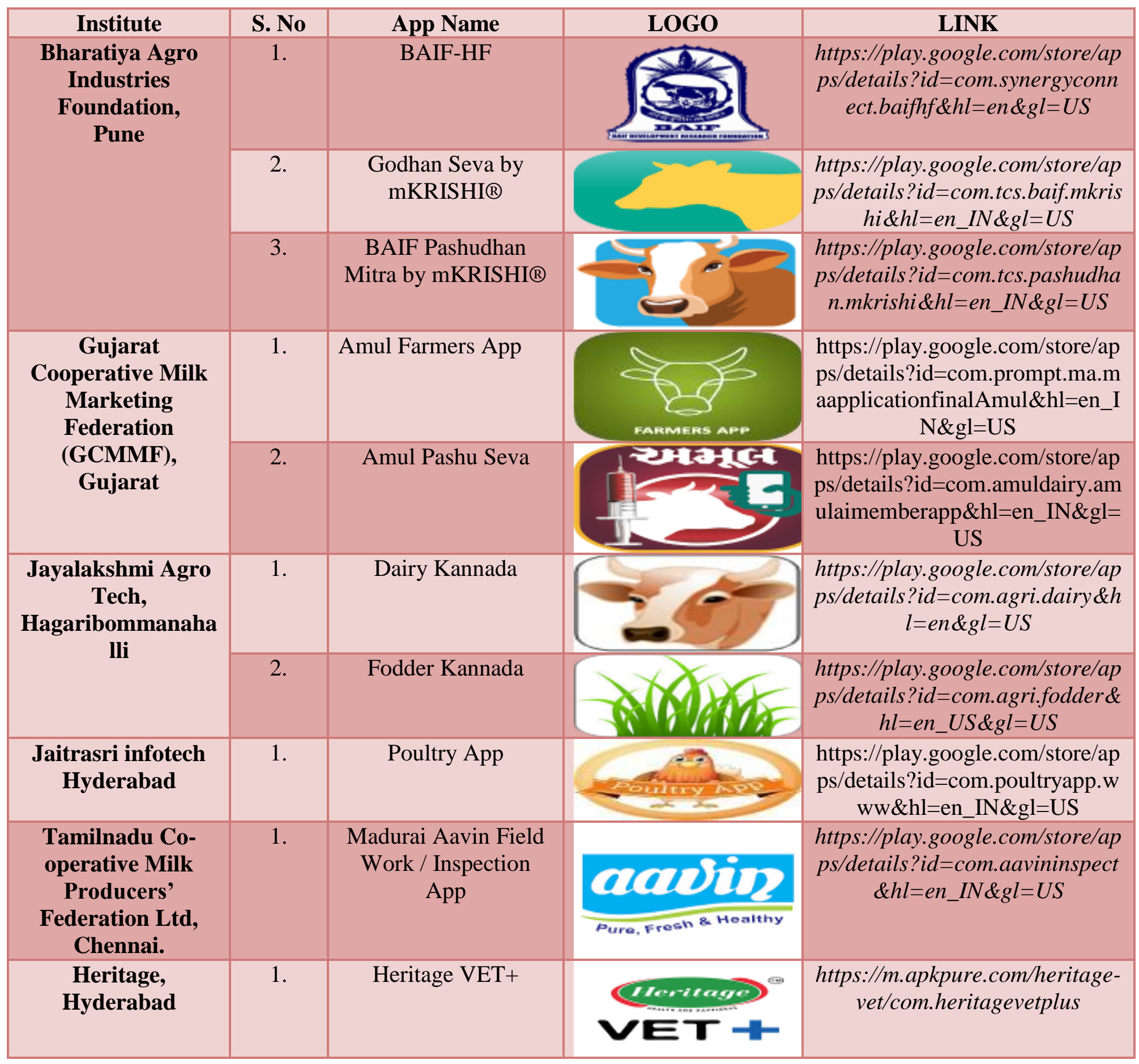




\begin{tabular}{|c|c|c|c|c|}
\hline $\begin{array}{l}\text { Mobility appz, } \\
\text { Indore. }\end{array}$ & 1. & $\begin{array}{l}\text { Krishi Vigyan in } \\
\text { Hindi } \\
\text { (किसान के लिए } \\
\text { उपयोगी) }\end{array}$ & कृषि चित्रान & $\begin{array}{c}\text { https://play.google.com/store/ap } \\
\text { ps/details?id=com.it.krishivigya } \\
n \& h l=e n \_I N\end{array}$ \\
\hline $\begin{array}{l}\text { Nithra Apps India } \\
\text { Private Limited, } \\
\text { Tamilnadu. }\end{array}$ & 1. & $\begin{array}{l}\text { Uzhavan Maadu - } \\
\text { உழவன் மாடு }\end{array}$ & & $\begin{array}{c}\text { https://play.google.com/store/ap } \\
\text { ps/details?id=nithra.tamil.madu } \\
\text { cattle.cow.breeding\&hl=ta\&gl } \\
=U S\end{array}$ \\
\hline $\begin{array}{c}\text { Wiki Kids Limited, } \\
\text { Hyderabad }\end{array}$ & 1. & Animal Husbandry & & $\begin{array}{l}\text { https://play.google.com/store/ap } \\
\text { ps/details?id=wwk.wikikids.com } \\
\text {.animalhusbandry\&hl=en_IN\& } \\
\text { gl=US }\end{array}$ \\
\hline $\begin{array}{l}\text { MJM Design, } \\
\text { Karnataka }\end{array}$ & 1. & $\begin{array}{l}\text { Animal Husbandry } \\
\text { Livestock }\end{array}$ & & $\begin{array}{c}\text { https://play.google.com/store/ap } \\
\text { ps/details?id=com.ls.livestock\& } \\
\text { hl=en\&gl=US }\end{array}$ \\
\hline Pashupalan & 1. & $\begin{array}{c}\text { Pashupalan } \\
\text { (पशुपालन/પशुપાલન) }\end{array}$ & & $\begin{array}{c}\text { https://play.google.com/store/ap } \\
\text { ps/details?id=com.animalhusba } \\
\text { ndry\&hl=gu\&gl=US }\end{array}$ \\
\hline $\begin{array}{l}\text { पशु मेला - Animall, } \\
\text { Karnataka }\end{array}$ & 1. & गाय भैंस wala पशुमेला & & $\begin{array}{c}\text { https://play.google.com/storelap } \\
\text { ps/details?id=in.animall.androi } \\
d \& h l=h i \& g l=U S\end{array}$ \\
\hline $\begin{array}{c}\text { कृषिफाई : } \\
\text { एग्रीकल्चर नेटवर्क } \\
\text { एप - मेड इन इंडिया, } \\
\text { Haryana }\end{array}$ & 1. & $\begin{array}{l}\text { Pashu Mela App - } \\
\text { Gaay Bhains ki } \\
\text { Kharid Bikri }\end{array}$ & & $\begin{array}{l}\text { https://play.google.com/storelap } \\
\text { ps/details?id=krishify.animal.pa } \\
\text { shu.mela\&hl=en_SG\&gl=US }\end{array}$ \\
\hline $\begin{array}{l}\text { Safasha Business } \\
\text { Solution's, } \\
\text { Lahore }\end{array}$ & 1. & $\begin{array}{l}\text { Cattle Market } \\
\text { Management System } \\
\text { (CMMS) }\end{array}$ & & $\begin{array}{c}\text { https://play.google.com/store/ap } \\
\text { ps/details?id=com.safashabusin } \\
\text { esssolution.cattlemarketcompan } \\
\text { y\&hl=en_US\&gl=US }\end{array}$ \\
\hline $\begin{array}{c}\text { Bigsys infotech pvt. } \\
\text { Ltd, } \\
\text { Uttar Pradesh }\end{array}$ & 1. & PASHU MANDI & & $\begin{array}{l}\text { https://play.google.com/store/ap } \\
\text { ps/details?id=com.pashu_mandi } \\
\text {.pashu_mela.pashu_bazaar.mar } \\
\text { ket\&hl=en_IN\&gl=US }\end{array}$ \\
\hline $\begin{array}{l}\text { Ma Narmada Dudh } \\
\text { Dairy, } \\
\text { Madhya Pradesh }\end{array}$ & 1. & Pashu Bazar & & $\begin{array}{l}\text { https://play.google.com/store/ap } \\
\text { ps/details?id=com.yogiksoft.pas } \\
\text { hu\&hl=en_IN\&gl=US }\end{array}$ \\
\hline $\begin{array}{c}\text { RNBV APPS, } \\
\text { Vijayawada }\end{array}$ & 1. & $\begin{array}{l}\text { Pig Master - A Guide } \\
\text { App for Pig Farming }\end{array}$ & & $\begin{array}{l}\text { https://play.google.com/storelap } \\
\text { ps/details?id=com.rnbvandroid } \\
\text { apps.pigapp\&hl=en_IN\&gl=US }\end{array}$ \\
\hline
\end{tabular}


Uneven digital access, low accuracy of data in apps, non-availability of content as per local need are important challenges in adopting mobile apps.

Numbers of agricultural applications are very limited compared to other sectors like health (Costopoulou et al., 2016).

Diversity of agricultural applications are limited in India compared to USA or Brazil (Barbosa et al., 2020).

There are digital tools which provide live services to various agricultural solutions with charge, which farmers unwilling to pay (Bonke et al., 2018)

\section{References}

Barbosa, J. Z., Prior, S. A., Pedreira, G. Q., Motta, A. C. V., Poggere, G. C., \& Goularte, G. D. (2020). Global trends in apps for agriculture. Multi-Science Journal (ISSN 2359-6902), 3(1), 1620.
Bonke, V., Fecke, W., Michels, M., \& Musshoff, O. (2018). Willingness to pay for smart phone apps facilitating sustainable crop protection. Agronomy for Sustainable Development, 38(5), 110.

Costopoulou, C., Ntaliani, M., \& Karetsos, S. (2016). Studying mobile apps for agriculture. IOSR J. Mob. Comput. Appl, 3(6), 44-49.

Inwood, S. E. E., \& Dale, V. H. (2019). State of apps targeting management for sustainability of agricultural landscapes. A review. Agronomy for sustainable development, 39(1), 8.

Rose, D. C., Sutherland, W. J., Parker, C., Lobley, M., Winter, M., Morris, C., \& Dicks, L. V. (2016).

Decision support tools for agriculture: Towards effective design and delivery. Agricultural systems, 149, 165-174. World Bank (2012).

Mobile Applications for rural development by Christine Zhenwei Qiang, Siou Chew Kuek, Andrew Dymond and Steve Esselaar.

\section{How to cite this article:}

Kakani Vijay Prakash, Debasis Ganguli, Arunasis Goswami and Chanchal Debnath. 2021. Animal Husbandry Mobile Apps in Transformation Livestock Farming. Int.J.Curr.Microbiol.App.Sci. 10(09): 518-530. doi: https://doi.org/10.20546/ijcmas.2021.1009.060 